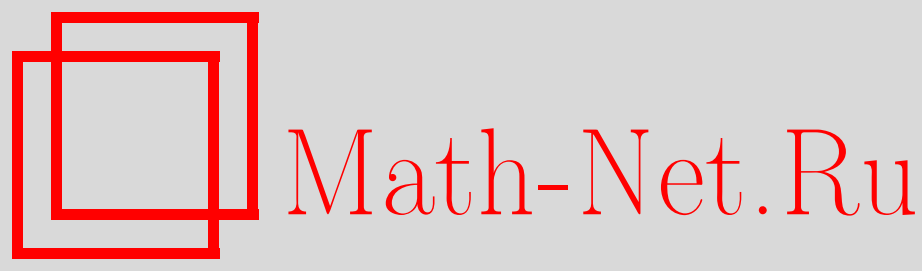

А. И. Бреев, И. В. Широков, А. А. Магазев, Поляризация вакуума скалярного поля на группах Ли и однородных пространствах, ТМФ, 2011, том 167, номер 1, 78-95

DOI: https://doi.org/10.4213/tmf6626

Использование Общероссийского математического портала Math-Net.Ru подразумевает, что вы прочитали и согласны с пользовательским соглашением http: //www . mathnet.ru/rus/agreement

Параметры загрузки:

IP: 18.234 .197 .8

26 апреля 2023 г., 16:02:24

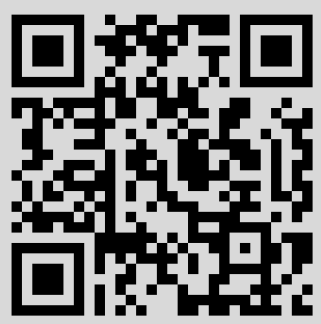




\title{
ПОЛЯРИЗАЦИЯ ВАКУУМА СКАЛЯРНОГО ПОЛЯ НА ГРУППАХ ЛИ И ОДНОРОДНЫХ ПРОСТРАНСТВАХ
}

\begin{abstract}
Предлагается метод вычисления вакуумных средних тензора энергии-импульса скалярного поля на группах Ли и однородных пространствах. Для решения поставленной задачи используется метод обобщенного гармонического анализа, базирующийся на методе орбит коприсоединенного представления.
\end{abstract}

Ключевые слова: поляризация вакуума, тензор энергии-импульса, гармонический анализ.

\section{1. ВВЕДЕНИЕ}

Исследование квантовых эффектов во внешних гравитационных полях является актуальной задачей современной квантовой теории поля (КТП). Основным объектом исследований выступает тензор энергии-импульса (ТЭИ), входящий в правую часть уравнений Эйнштейна и описывающий взаимодействие материи и гравитации. В связи с этим важное значение приобретает задача вычисления вакуумных средних ТЭИ, которые позволяют учесть обратное влияние квантового поля на геометрию пространства-времени в рамках однопетлевого приближения к полностью квантованной теории (КТП в искривленном пространстве-времени). Отметим, что к настоящему времени теория поля в искривленном пространстве-времени разработана достаточно хорошо, и ей посвящена обширная литература (см. книги [1]-[3]).

Для получения конечных значений вакуумных средних ТЭИ необходимо проведение процедуры регуляризации. Существует несколько различных методов, эффективное применение которых зависит от конкретной задачи. В случае, когда пространство однородно и изотропно, эффективно применение метода размерной регуляризации [4]. Другой способ регуляризации - метод раздвижения аргументов операторов поля в билинейной форме ТЭИ, предложенный в работе [5]. Стоит

\footnotetext{
*Томский государственный университет, Томск, Россия.

E-mail: alphmu2856@rambler.ru

${ }^{\dagger}$ Омский государственный технический университет, Омск, Россия. E-mail: iv_shirokov@mail.ru

${ }^{\ddagger}$ Иртышский филиал ФГОУ ВПО "Новосибирская государственная академия водного транспорта", Омск, Россия. E-mail: magazev@mail.ru
} 
отметить, что, хотя вышеупомянутые методы регуляризации не требуют расчета вакуумных средних ТЭИ, расчет последних также имеет значение, так как в ряде случаев возможно избавиться от расходимости напрямую (например, при помощи метода $n$-волновой регуляризации [6]).

Настоящая работа посвящена вычислению вакуумных средних ТЭИ скалярного поля на псевдоримановых многообразиях вида $\mathbb{R}^{1} \times G$ и $\mathbb{R}^{1} \times P$, где $G$ - локальная группа Ли с правоинвариантной метрикой, а $P$ - правое однородное пространство с $G$-инвариантной метрикой и нулевым дефектом, причем случай однородного пространства сводится к расчетам на групповом многообразии $\mathbb{R}^{1} \times G$, где $G$ - группа преобразований однородного пространства.

Интерес к этой проблеме связан с тем, что практически все известные в настоящее время модели римановых многообразий общей теории относительности связаны с различными группами преобразований и нередко относятся к классу однородных римановых пространств. К примеру, имеется много работ, посвященных исследованию квантовых эффектов в пространстве де Ситтера (упомянем работы [7], [8]).

В настоящей работе для решения поставленной задачи применяется метод орбит. Метод орбит коприсоединенного представления (К-орбит) впервые был описан в работах [9], [10]. Основные результаты метода приведены в работах [11]-[13].

При помощи метода орбит проводится интегрирование уравнения Клейна-Гордона (так называемый метод некоммутативного интегрирования [14], [15]). В отличие от широко известного метода разделения переменных [16], данный метод учитывает некоммутативную алгебру операторов симметрии уравнения и позволяет проводить интегрирование в случае, когда провести разделение переменных не представляется возможным. Причем решение строится глобально, и не возникает проблемы сшивки решений в различных картах атласа исходного многообразия, так как исходное уравнение редуцируется к уравнению на лагранжевом подмногообразии к невырожденной К-орбите, обладающей более простой геометрией и топологией.

Полученное в настоящей работе выражение для вакуумных средних ТЭИ скалярного поля не зависит от локальных координат и выражается через операторы $\lambda$-представления алгебры Ли группы Ли и решения дифференциального уравнения на К-орбите.

Также в работе получено выражение для обобщенной дзета-функции оператора уравнения Клейна-Гордона через величины, определенные на невырожденной $\mathrm{K}$-орбите группы Ли $G$, а не на исходном групповом многообразии. Это облегчает нахождение аналитического продолжения дзета-функции в конкретных задачах, так как выражение получается более простым и зависит от меньшего количества переменных.

В связи с этим для случая группового многообразия адаптирован прямой метод обобщенной дзета-функции, развитый в работе [17]. Предлагаемый нами метод перенормировки вакуумных средних ТЭИ основан на вычислении функциональных производных от однопетлевого эффективного действия по метрике и сводит задачу к поиску аналитического продолжения для обобщенной дзета-функции.

При помощи предлагаемой в настоящей работе техники вычисления вакуумных средних ТЭИ проведен расчет эффекта Казимира на групповом многообразии вида $\mathbb{R} \times E(2)$, где $E(2)$ - группа движений двумерной плоскости, оснащенная правоинвариантной метрикой наиболее общего вида. Заметим, что в этом случае использовать метод разделения переменных не представляется возможным. 


\section{G-ИНВАРИАНТНЫЕ МЕТРИКИ НА ОДНОРОДНЫХ ПРОСТРАНСТВАХ}

Пусть $G$ - связная односвязная унимодулярная вещественная группа Ли, $L-$ алгебра Ли группы Ли $G, L^{*}$ - пространство линейных функционалов на $L, \mathcal{X}(G)-$ линейное пространство гладких векторных полей на группе Ли $G ; \xi_{X}(x)=\left(L_{x}\right)_{*} X$, $\eta_{X}(x)=-\left(R_{x}\right)_{*} X, X \in L, x \in G,-$ лево- и правоинвариантные векторные поля на группе Ли $G$ (генераторы соответственно правых и левых сдвигов).

Пусть $\mathbf{G}$ - невырожденная квадратичная форма на алгебре Ли $L$, задающая метрику на группе Ли $G$ в единице. При помощи правых сдвигов введем на группе Ли $G$ правоинвариантную метрику $\langle\cdot, \cdot\rangle_{x}$ в произвольной точке $x \in G$ :

$$
\langle\sigma, \tau\rangle_{x}=\mathbf{G}\left(\left(R_{x}^{-1}\right)_{*} \sigma,\left(R_{x}^{-1}\right)_{*} \tau\right), \quad \sigma, \tau \in T_{x} G .
$$

Равенство (1), записанное в локальных координатах, дает выражение для метрического тензора $\gamma_{i j}(x)$ на группе Ли $G, i, j=1, \ldots, \operatorname{dim} L$ :

$$
\gamma_{i j}(x)=\gamma_{A B} \sigma_{i}^{A}(x) \sigma_{j}^{B}(x), \quad \gamma_{A B} \equiv \mathbf{G}\left(e_{A}, e_{B}\right),
$$

где $\left\{e_{A}\right\}$ - некоторый фиксированный базис в алгебре $L, \sigma^{B}(x)$ - базисные правоинвариантные 1-формы: $\sigma^{B}(x) \equiv\left(R_{x}\right)^{*} e^{B},\left\{e^{B}\right\}-$ базис в $L^{*}:\left\langle e_{A}, e^{B}\right\rangle=\delta_{A}^{B}$, $A, B=1, \ldots, \operatorname{dim} L$.

Существует единственная симметричная и согласованная с метрикой (1) связность Леви-Чивита $\nabla$ на группе Ли $G$. Можно показать, что связность $\nabla$ является правоинвариантной и однозначно определяется билинейным отображением $\Gamma: L \times L \rightarrow L:$

$$
\begin{gathered}
\nabla_{\eta_{X}} \eta_{Y}=\left(R_{x}\right)_{*} \boldsymbol{\Gamma}(X, Y), \\
\boldsymbol{\Gamma}(X, Y)=\frac{1}{2}\left([X, Y]-\operatorname{ad}_{X}^{*} Y-\operatorname{ad}_{Y}^{*} X\right), \quad X, Y \in L .
\end{gathered}
$$

Тензор кривизны Римана, построенный по связности (2), в свою очередь однозначно определяется отображением $\mathbf{R}(X, Y): L \rightarrow L$ :

$$
\mathbf{R}(X, Y) Z=\boldsymbol{\Gamma}(Y, \boldsymbol{\Gamma}(X, Z))-\boldsymbol{\Gamma}(X, \boldsymbol{\Gamma}(Y, Z))+\boldsymbol{\Gamma}([X, Y], Z), \quad X, Y, Z \in L,
$$

и на произвольных векторных полях $\tau, v, \zeta \in \mathcal{X}(G)$ принимает значение

$$
R(\tau, v) \zeta=\left(R_{x}\right)_{*} \mathbf{R}\left(\left(R_{x}^{-1}\right)_{*} \tau,\left(R_{x}^{-1}\right)_{*} v\right)\left(R_{x}^{-1}\right)_{*} \zeta
$$

Компоненты символов Кристоффеля $\Gamma_{A B}^{C} \equiv \mathbf{G}\left(e^{C}, \boldsymbol{\Gamma}\left(e_{A}, e_{B}\right)\right)$ выражаются через компоненты метрики $\gamma_{A B}$ и структурные константы алгебры Ли $L$ :

$$
\Gamma_{A B}^{C}=\frac{1}{2} \gamma^{C D}\left(C_{A D B}+C_{B D A}-C_{D A B}\right), \quad C_{A B C} \equiv \gamma_{A D} C_{B C}^{D} .
$$

Приведем выражение для компонент тензора Риччи $R_{C B} \equiv R\left(\eta_{C}, \eta_{B}\right)$ в базисе правоинвариантных векторных полей:

$$
R_{C B}=\Gamma_{B A}^{E} \Gamma_{D E}^{A}-C_{E A}^{A} \Gamma_{B D}^{E} .
$$


Пусть группа $G$ эффективно действует на однородном многообразии $P$ при помощи правых сдвигов. Обозначим через $H$ замкнутую стационарную подгруппу некоторой точки $y_{0} \in P$, через $\mathfrak{h}$ - алгебру Ли группы $H$. В этом случае $P$ диффеоморфно фактор-многообразию $G / H$ правых смежных классов группы Ли $G$, и многообразию $P$ естественно сопоставляется главное расслоение $G(P, H, \pi)$.

Над областями тривиализации $U \in P$ в расслоенном пространстве $G$ введем координаты $x^{A}=\left(y^{a}, h^{\alpha}\right)$ прямого произведения $U \times H(a=1, \ldots, \operatorname{dim} P, \alpha=$ $\operatorname{dim} P+1, \ldots, \operatorname{dim} G)$. При этом координаты произвольной точки $x \in G$ можно представить в виде $x=h s(y)$, где $s: P \rightarrow G$ - локальное гладкое сечение расслоения $G$. В свою очередь линейное пространство алгебры Ли $L$ допускает разложение в прямую сумму подпространств $L=\mathfrak{h} \oplus \mathfrak{m}$, где $\mathfrak{m}$ - дополнение к $\mathfrak{h}$.

Обозначим через $\mathcal{X}(P)$ линейное пространство гладких векторных полей на однородном пространстве $P$. Векторное поле $\tau \in \mathcal{X}(G)$ будем называть проектируемым на однородное пространство $P$, если при поточечной проекции $\pi_{*}: T_{x} G \rightarrow T_{\pi(x)} P$ векторное поле $\tau$ переходит в некоторое векторное поле $\pi_{*}(\tau) \in \mathcal{X}(P)$. Для того чтобы векторное поле $\tau$ на группе Ли $G$ было проектируемым, необходимым и достаточным является выполнение условия $\pi_{*}\left(\left[\eta_{Y}, \tau\right]\right)=0, Y \in \mathfrak{h}$. Множество проектируемых векторных полей образует подалгебру $\mathcal{B}(G)$ в $\mathcal{X}(G)$, и имеет место изоморфизм $\mathcal{X}(P)=\mathcal{B}(G) / \operatorname{ker}\left\{\pi_{*}\right\}$.

Рассмотрим $G$-инвариантную метрику на однородном пространстве $P$, построенную по соответствующей правоинвариантной метрике (1) на группе Ли $G$. А именно, наложим на квадратичную форму $\mathbf{G}$ условие

$$
\mathbf{G}([X, Y], Z)+\mathbf{G}(Y,[X, Z])=0, \quad X \in \mathfrak{h}, \quad Y, Z \in L,
$$

определим на алгебре Ли $L$ квадратичную форму $\mathbf{B}$ :

$$
\begin{gathered}
\operatorname{ker} \mathbf{B}=\mathfrak{h}, \\
\mathbf{B}(X, Y)=\mathbf{G}(X, Y), \quad X, Y \in \mathfrak{m},
\end{gathered}
$$

и положим по определению

$$
\left\langle\pi_{*}(\tau), \pi_{*}(v)\right\rangle_{\pi(x)} \equiv \mathbf{B}\left(\left(R_{x}^{-1}\right)_{*} \sigma,\left(R_{x}^{-1}\right)_{*} \tau\right), \quad v, \tau \in \mathcal{B}(G) .
$$

Условие (1) обеспечивает независимость определения метрики от выбора векторных полей $\tau, v \in \mathcal{B}(G)$ таких, что $\pi_{*}(\tau)=\pi_{*}(v)$. Соотношение (4) эквивалентно требованию $\operatorname{Ad}(H)$-инвариантности формы $B$ и обеспечивает независимость метрики при действии группы $H$ левыми сдвигами на пространстве расслоения $G$. Очевидно, что любой $G$-инвариантной метрике на $P$ может быть сопоставлен класс правоинвариантных метрик на группе Ли $G$, и любая $G$-инвариантная метрика на $P$ может быть построена данным образом.

Можно показать, что символы Кристоффеля связности Леви-Чивита, построенной по $G$-инвариантной метрике, имеют вид

$$
\Gamma_{j k}^{i}(y)=\Gamma_{b c}^{a} \sigma_{j}^{b}(x) \sigma_{k}^{c}(x) \eta_{a}^{i}(x)-\sigma_{j}^{b}(x) \eta_{b, k}^{i}-C_{b \alpha}^{a} \sigma_{j}^{b}(x) \sigma_{k}^{\alpha}(x) \eta_{a}^{i}(x),
$$

где величины $\Gamma_{b c}^{a}$ не зависят от координат и определяются компонентами $G^{a b}$ квадратичной формы $\mathbf{G}$ и структурными константами алгебры Ли $L$ :

$$
\Gamma_{b c}^{a}=-\frac{1}{2} C_{b c}^{a}-\frac{1}{2} G^{a d}\left[G_{e c} C_{b d}^{e}+G_{e b} C_{c d}^{e}\right]
$$


Тензор Римана связности Леви-Чивита на однородном пространстве также выражается через $\Gamma_{b c}^{a}$, базисные правоинвариантные 1-формы и векторные поля на группе Ли $G$ :

$$
\begin{aligned}
R_{j k l}^{i}(y) & =R_{b c d}^{a} \sigma_{i}^{b}(x) \sigma_{k}^{c}(x) \sigma_{l}^{d}(x) \eta_{a}^{i}(x), \\
R_{b c d}^{a} & =\Gamma_{\bar{d} d}^{a} \Gamma_{b c}^{\bar{d}}-\Gamma_{\bar{d} c}^{a} \Gamma_{b d}^{\bar{d}}+C_{c d}^{\bar{c}} \Gamma_{b \bar{c}}^{a} .
\end{aligned}
$$

\section{3. К-ОРБИТЫ. ГАРМОНИЧЕСКИЙ АНАЛИЗ}

Группа Ли $G$ действует на сопряженном пространстве $L^{*}$ коприсоединенным представлением $\mathrm{Ad}^{*}: G \times L^{*} \rightarrow L^{*},(x, f) \rightarrow \operatorname{Ad}_{x}^{*} f$ по правилу

$$
\left\langle\operatorname{Ad}_{x}^{*} f, X\right\rangle=\left\langle f, \operatorname{Ad}_{x^{-1}} X\right\rangle, \quad f \in L^{*}, \quad X \in L, \quad x \in G,
$$

где $\operatorname{Ad}_{x}-$ присоединенное действие группы Ли $G$ на $L$. На дуальном пространстве $L^{*}$ определена скобка Пуассона-Ли

$$
\{\phi, \psi\}^{\mathrm{Lie}}(f) \equiv\langle f,[\nabla \phi(f), \nabla \psi(f)]\rangle, \quad \phi, \psi \in C^{\infty}\left(L^{*}\right), \quad f \in L^{*} .
$$

В силу вырожденности скобки (8) на $L^{*}$ существуют функции Казимира $K_{\mu}(f)$, коммутирующие со всеми функциями из $C^{\infty}\left(L^{*}\right)$ и являющиеся инвариантами коприсоединенного представления: $K_{\mu}\left(\operatorname{Ad}_{x}^{*} f\right)=K_{\mu}(f), x \in G$. Число независимых функций Казимира ind $L$ называется индексом алгебры $L: \mu=1, \ldots$, ind $L$.

Коприсоединенное действие $\mathrm{Ad}^{*}$ расслаивает $L^{*}$ на K-орбиты. Размерность отдельной $\mathrm{K}$-орбиты равна $\operatorname{dim} L-\operatorname{ind} L-2 k$, где $k=0, \ldots,(\operatorname{dim} L-\operatorname{ind} L) / 2$. Коалгебра $L^{*}$ является объединением связных инвариантных алгебраических поверхностей $M_{(s)}$, где каждая связная поверхность $M_{(s)}$ является объединением К-орбит размерности $\operatorname{dim} G-$ ind $L-2 s$.

Непостоянные на $M_{(s)}$ функции $K_{\mu}^{(s)}(f)$, коммутирующие с любой функцией на $M_{(s)}$, называются функииями Казимира $s$-типа. Через $r_{(s)}$ обозначим количество функционально независимых функций Казимира $s$-типа, причем $\operatorname{dim} M_{(s)}=r_{(s)}$. К-орбита называется орбитой $s$-типа, если $\mathcal{O}_{\lambda} \in M_{(s)}$, а число $s$ - степенъю върождения орбитъ. К-орбиты с нулевой степенью вырождения называются невырожденными, а остальные сингулярными. Через $F_{\alpha}^{(s)}(f), \alpha=1, \ldots, \operatorname{dim} L-r_{(s)}$, обозначим независимый набор функций, определяющих поверхность $M_{(s)}$.

Пусть далее $\mathcal{O}_{\lambda}-\mathrm{K}$-орбита группы Ли $G s$-типа, содержащая ковектор $\lambda$. Введем на $\mathcal{O}_{\lambda}$ замкнутую невырожденную 2-форму $\omega_{\lambda}$, действующую на касательных к K-орбите векторах $a, b \in T_{\lambda} \mathcal{O}_{\lambda}$, следующим образом:

$$
\omega_{\lambda}(a, b)=\langle\lambda,[\alpha, \beta]\rangle, \quad a=\operatorname{ad}_{\alpha}^{*} \lambda, \quad b=\operatorname{ad}_{\beta}^{*} \lambda, \quad \alpha, \beta \in L .
$$

2-Форма (9) называется формой Кириллова и задает на K-орбите $\mathcal{O}_{\lambda}$ симплектическую структуру. Ограничение скобки Пуассона (8) на К-орбиту совпадает со скобкой Пуассона, порожденной симплектической формой $\omega_{\lambda}$. Известно, что на симплектическом многообразии существуют канонические координаты Дарбу, в которых симплектическая форма имеет канонический вид. Обозначим через $(p, q) \in P \times Q$ канонические координаты Дарбу на К-орбите $\mathcal{O}_{\lambda}$, в которых форма Кириллова $\omega_{\lambda}$ принимает канонический вид: $\omega_{\lambda}=d p_{a} \wedge d q^{a}, a=1, \ldots, \operatorname{dim} \mathcal{O}_{\lambda} / 2$. Заметим, что области $P$ и $Q$ изменения канонических координат $(p, q)$ являются лагранжевыми подмногообразиями к K-орбите $\mathcal{O}_{\lambda}$. 
Определим каноническое вложение $f: \mathcal{O}_{\lambda} \rightarrow L^{*}$, когда ковектору $f \in L^{*}$ ставятся в соответствие его канонические координаты на $\mathrm{K}$-орбите $\mathcal{O}_{\lambda}$, содержащей ковектор $f$. Каноническое вложение однозначно определяется функциями $f_{X}=$ $f_{X}(p, q, \lambda), X \in L$, удовлетворяющими системе уравнений

$$
\left\{f_{X}, f_{Y}\right\}^{\text {Lie }}=f_{[X, Y]}, \quad f_{X}(0,0, \lambda)=\lambda(X), \quad X, Y \in L .
$$

Так как $f \in M_{(s)}$, то в случае сингулярных К-орбит каноническое вложение также должно удовлетворять условию $F_{\alpha}^{(s)}(f)=0, \alpha=1, \ldots, \operatorname{dim} L-r_{(s)}$. Рассмотрим важный частный случай, когда каноническое вложение (10) линейно по переменным $p$ :

$$
f_{X}(q, p, \lambda)=\alpha_{X}^{a}(q) p_{a}+\chi_{X}(q, \lambda), \quad X \in L, \quad a=1, \ldots, \operatorname{dim} \mathcal{O}_{\lambda} / 2
$$

Можно показать, что для существования линейного канонического вложения (11) орбиты $\mathcal{O}_{\lambda}$ необходимо и достаточно, чтобы функционал $\lambda$ допускал поляризацию $\mathbf{n}$. Напомним, что поляризацией $\mathbf{n}$ функционала $\lambda$ называется подалгебра размерности $\operatorname{dim} \mathbf{n}=\operatorname{dim} L-\operatorname{dim} \mathcal{O}_{\lambda} / 2$, подчиненная функционалу $\lambda:\langle\lambda,[\mathbf{n}, \mathbf{n}]\rangle=0$.

Операторы $l_{X}(q, \lambda) \equiv i f_{X}\left(q,-i \partial_{q}, \lambda\right)$ реализуют неприводимое представление алгебры Ли $L$ в пространстве гладких функций $L(Q, \mathbf{n}, \lambda)$ от $\operatorname{dim} Q=\operatorname{dim} \mathcal{O}_{\lambda} / 2$ переменных. Будем называть данное представление $\lambda$-представлением алгебры Ли $L$ $s$-типа.

Введем на многообразии $Q$ меру $d \mu(q)$ и скалярное произведение

$$
\left(\psi_{1}, \psi_{2}\right)=\int_{Q} \overline{\psi_{1}(q)} \psi_{2}(q) d \mu(q)
$$

Потребуем, чтобы операторы $\lambda$-представления были косоэрмитовы относительно (12). Для выполнения этого условия необходимо и достаточно ввести "квантовый сдвиг" $\lambda \rightarrow \lambda+i \beta$, где параметр $\beta$ определяется выражением

$$
\beta(X)=\frac{1}{2}\left[\operatorname{tr}\left(\operatorname{ad}_{X}\right)-\operatorname{tr}\left(\operatorname{ad}_{X} \mid \mathfrak{r}\right)\right]
$$

где $\mathfrak{l}$ - вещественное подпространство, построенное по комплексной поляризации: $\mathfrak{l}=\mathfrak{n}+\overline{\mathfrak{n}}$. Формула (13) справедлива в случае, если $\mathfrak{l}-$ подалгебра.

Введем поднятие $\lambda$-представления алгебры Ли $L$ до локального представления ее группы Ли $G$ :

$$
\begin{aligned}
T^{\lambda}(x) \varphi(q) & =\int D_{q \bar{q}^{\prime}}^{\lambda}(x) \varphi\left(q^{\prime}\right) d \mu\left(q^{\prime}\right), \\
\left.\frac{\partial}{\partial t}\right|_{t=0} T^{\lambda} e^{t X} \varphi(q) & =l_{X}(q, \lambda) \varphi(q),
\end{aligned}
$$

где $\varphi \in C^{\infty}(Q)$. Из условия $T^{\lambda}\left(x_{1}\right) T^{\lambda}\left(x_{2}\right)=T^{\lambda}\left(x_{1} x_{2}\right)$ следует соотношение для "матричных" элементов представления $T^{\lambda}$ :

$$
D_{q \bar{q}^{\prime}}^{\lambda}\left(x_{1} x_{2}\right)=\int D_{q \bar{q}^{\prime \prime}}^{\lambda}\left(x_{1}\right) D_{q^{\prime \prime} \bar{q}^{\prime}}^{\lambda}\left(x_{2}\right) d \mu\left(q^{\prime \prime}\right)
$$


Если представление $T^{\lambda}(x)$ унитарно: $l_{X}^{\dagger}=-l_{X}$, то $D_{q \bar{q}^{\prime}}^{\lambda}(x)=\overline{D_{q^{\prime} \bar{q}}^{\lambda}\left(x^{-1}\right)}$. Можно показать, что обобщенные функции $D_{q \bar{q}^{\prime}}^{\lambda}(x)$ удовлетворяют переопределенной системе уравнений

$$
\left[\eta_{X}(x)+l_{X}(q, \lambda)\right] D_{q \bar{q}^{\prime}}^{\lambda}(x)=0, \quad\left[\xi_{X}(x)-\overline{l_{X}^{\dagger}\left(q^{\prime}, \lambda\right)}\right] D_{q \bar{q}^{\prime}}^{\lambda}(x)=0 .
$$

Из требования однозначной определенности функций $D_{q \bar{q}^{\prime}}^{\lambda}(x)$ на группе Ли $G$ следует условие Кириллова целочисленности орбиты $\mathcal{O}_{\lambda}[11]$ :

$$
\frac{1}{2 \pi} \int_{\gamma \in H_{2}\left(\mathcal{O}_{\lambda}\right)} \omega_{\lambda}=n_{\gamma} \in \mathbb{Z},
$$

где $\omega_{\lambda}$ - форма Кириллова на К-орбите.

Семейство обобщенных функций $D_{q \bar{q}^{\prime}}^{\lambda}(x)$ полно и ортогонально, и для каждой функции $\phi(x)$ из плотного ядерного подпространства

$$
L_{(s)}=\left\{\phi \in L_{2}(G, d x) \mid F_{\alpha}^{(s)}\left(\xi_{X}(x) \phi(x)\right)=0, X \in L\right\}
$$

определены прямое и обратное преобразования Фурье:

$$
\begin{aligned}
\psi\left(q, q^{\prime}, \lambda\right) & =\int \phi(x) \overline{D_{q \bar{q}^{\prime}}^{\lambda}}\left(x^{-1}\right) d \mu(x), \\
\phi(x) & =\int \psi\left(q, q^{\prime}, \lambda\right) D_{q \bar{q}^{\prime}}^{\lambda}\left(x^{-1}\right) d \mu(q) d \mu\left(q^{\prime}\right) d \mu(\lambda),
\end{aligned}
$$

где $d \mu(\lambda)$ - спектральная мера операторов Казимира $K_{\mu}(\eta)$. Для невырожденных орбит прямое и обратное преобразования $(17),(18)$ определены на всем пространстве $L_{2}(G, d x)$.

Отметим, что если функции $\varphi(x)$ и $\psi\left(q, q^{\prime}, \lambda\right)$ преобразуются друг в друга согласно преобразованиям (17), (18), то выполняются соотношения дуальности:

$$
\eta_{X}(x) \Leftrightarrow l_{X}\left(q^{\prime}, \lambda\right), \quad \xi_{X}(x) \Leftrightarrow \overline{l_{X}^{\dagger}(q, \lambda)},
$$

т.е. образы дифференциальных операторов $\xi_{X}(x), \eta_{X}(x)$ при преобразовании (17) являются дифференциальными операторами, действующими в пространстве $Q$.

Кратко опишем процедуру построения гармонического анализа на однородном пространстве $P$, следуя работам [18], [19]. Суть метода состоит в том, чтобы свести гармонический анализ на однородном пространстве $P$ к гармоническому анализу на группе Ли $G$, изложенному выше, при наложении дополнительных условий.

Любую функцию $\widehat{\varphi} \in C^{\infty}(P)$ на однородном пространстве $P$ можно отождествить с функцией $\varphi \in C^{\infty}(G)$, постоянной на правых классах смежности $H x$. Имеет место изоморфизм $C^{\infty}(P) \sim \mathcal{F}$, где функциональное подпространство $\mathcal{F}$ функций, постоянных на слое расслоения $G(P, H, \pi)$, ввиду связности группы Ли $H$ определяется следующим образом:

$$
\mathcal{F} \equiv\left\{\varphi \in C^{\infty}(G) \mid \eta_{X} \varphi(x)=0, X \in \mathfrak{h}, x \in G\right\} .
$$

Определим функциональное подпространство $L_{(s)}(P)$ пространства $L_{2}(P, d \mu(y))$ как проекцию пространств $L_{(s)}$ :

$$
L_{(s)}(P)=L_{(s)} \cap \mathcal{F}=\left\{\varphi \in L_{2}(P, d \mu(y)) \mid F^{(s)}(\tilde{X}) \varphi=0\right\},
$$


где $\widetilde{X}$ - генераторы группы преобразований Ли $G$, действующей на $P$. В работе [18] показано, что пространства $L_{(s)}(P)$ при $s<s_{P}$ пустые и

$$
F^{\left(s_{P}\right)}(\widetilde{X}) \equiv 0, \quad \widetilde{K}_{\mu}^{\left(s_{P}\right)}(i \widetilde{X}) \equiv 0, \quad \mu=1, \ldots, r_{s_{P}},
$$

где $s_{P}$ - число такое, что $M_{\left(s_{P}\right)} \cap \mathfrak{h}^{\perp} \neq \varnothing$, но $M_{\left(s_{P}-1\right)} \cap \mathfrak{h}^{\perp}=\varnothing$. Данное число называется степенъю вырождения однородного пространства $P ; \widetilde{K}_{\mu}^{\left(s_{P}\right)}(f)$ - функции Ка-

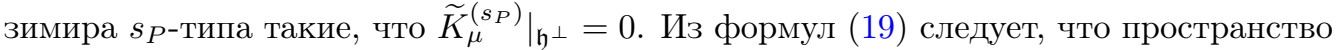
$L_{\left(s_{P}\right)}(P)$ совпадает с пространством $L_{2}(P, d \mu(y))$, и, следовательно, гармонический анализ на однородном пространстве сводится к гармоническому анализу в пространстве $L_{\left(s_{P}\right)}$ функций на группе Ли $G$, подчиненных условию $\varphi \in \mathcal{F}$.

Будем говорить, что $\lambda$-представление алгебры $L$ coответствует однородному пространству $P$, если

$$
\widetilde{K}_{\mu}^{\left(s_{P}\right)}(\lambda(j))=0, \quad \mu=1, \ldots, r_{s_{P}}
$$

Поднимем $\lambda$-представление алгебры $L s_{P}$-типа до соответствующего представления группы Ли $G$ в пространстве функций $\mathcal{F}$. Можно показать, что в этом случае набор функций $D_{q \bar{q}^{\prime}}^{\lambda}(x)$ является полным и ортогональным и образует базис функционального пространства $L_{\left(s_{P}\right)}$. Спроектируем функциональное пространство $L_{\left(s_{P}\right)}$ на пространство функций $L_{2}(P, d \mu(y))$. Для согласования с тождествами (19) необходимо наложить условия (20), т.е. $\lambda$-представление должно соответствовать однородному пространству. В этом случае точно определены прямое и обратное преобразования Фурье $(17),(18)$, но на функции $\psi\left(q, q^{\prime}, \lambda\right)$ (в силу того, что $\phi \in \mathcal{F}$ ) необходимо наложить требование

$$
l_{\alpha}\left(q^{\prime}, \lambda\right) \psi\left(q, q^{\prime}, \lambda\right)=0, \quad \alpha=1, \ldots, \operatorname{dim} H
$$

Целое неотрицательное число

$$
d_{P}=\frac{1}{2} \sup \operatorname{rank}\langle\lambda,[L, L]\rangle-\sup \operatorname{rank}\langle\lambda,[L, \mathfrak{h}]\rangle, \quad \lambda \in \mathfrak{h}^{\perp},
$$

называется дефектом однородного пространства Р. Пространствами нулевого дефекта являются симметрические пространства, евклидовы пространства. Для пространств нулевого дефекта алгебра инвариантных операторов на однородном пространстве коммутативна, и система (21) полностью разрешима. В противном случае алгебра инвариантных операторов нетривиальна, и построение гармонического анализа требует введения специальных конструкций (см. работу [18]).

\section{4. ИНТЕГРИРОВАНИЕ УРАВНЕНИЯ КЛЕЙНА-ГОРДОНА}

Рассмотрим $(n+1)$-мерное групповое многообразие $M^{n+1}=\mathbb{R}^{1} \times G^{n}$, где элемент группы $g \in M$ будем обозначать как $(x, t), x \in G, t \in \mathbb{R}$. Метрический тензор на $M$ выберем в виде $\Theta_{A^{\prime} B^{\prime}}=\left(1 \oplus-\gamma_{A B}\right)$, где $\gamma_{A B}$ - тетрадные компоненты правоинвариантной метрики на группе Ли $G$, индексы вида $A^{\prime}, B^{\prime}, \ldots=0,1, \ldots, n$ обозначают тетрадные компоненты на $M$, а индексы $A, B, \ldots=1, \ldots, n$ - тетрадные компоненты на группе Ли $G$, причем $\xi_{0}=-\eta_{0}=\partial_{t}$. 
Рассмотрим комплексное скалярное поле $\varphi(x, t) \in C^{\infty}(M)$ массы $m$ с лагранжианом

$$
\mathcal{L}^{0}=\Theta^{A^{\prime} B^{\prime}} \eta_{A^{\prime}} \overline{\varphi(x, t)} \eta_{B^{\prime}} \varphi(x, t)-\left[m^{2}+\zeta R\right]|\varphi(x, t)|^{2},
$$

где $\zeta=(n-1) /(4 n)$ - конформная связь с кривизной, $R$ - скалярная кривизна многообразия $M$. В качестве уравнения Эйлера-Лагранжа для действия с лагранжианом (22) имеем уравнение Клейна-Гордона

$$
\left(\partial_{t}^{2}-\Delta_{G}+\zeta R+m^{2}\right) \varphi(x, t)=0
$$

где $\Delta_{G}=\gamma^{A B} \eta_{A} \eta_{B}$ - оператор Лапласа на группе Ли $G$.

Построим базис решений $\varphi_{\sigma}(x, t)$ уравнения $(23)$, нумеруемый коллективным индексом $\sigma$ и удовлетворяющий условию нормировки

$$
-i \int \varphi_{\sigma}(x, t) \overleftrightarrow{\partial_{t}} \varphi_{\sigma^{\prime}}(x, t) d \mu(x)=\delta\left(\sigma, \sigma^{\prime}\right)
$$

Пусть $\varphi(x, t)=f(t) F(x)$. Тогда

$$
f_{\Lambda}(t)=\frac{1}{\sqrt{2 \omega}} e^{-i \omega t}, \quad \omega^{2}=\Lambda^{2}+\zeta R+m^{2},
$$

где $\Lambda$ - параметр разделения переменных. Для функции $F(x)$ имеем уравнение Клейна-Фока на группе Ли $G$ :

$$
-\Delta_{G} F_{\Lambda}(x)=\Lambda^{2} F_{\Lambda}(x)
$$

При помощи преобразования (17) редуцируем уравнение (26) к уравнению на невырожденной К-орбите. При этом оператор Лапласа $\Delta_{G}$ перейдет в дифференциальный оператор $H\left(l\left(q^{\prime}, \lambda\right)\right)=\gamma^{A B} l_{A}\left(q^{\prime}, \lambda\right) l_{B}\left(q^{\prime}, \lambda\right)$, действующий на лагранжевом подмногообразии $Q$ к K-орбите $\mathcal{O}_{\lambda}$ от $\operatorname{dim} Q=\operatorname{dim} \mathcal{O}_{\lambda} / 2$ переменных $q$.

Из формулы (17) следует, что переменные $q$ будут входить в уравнение на K-орбите как параметры, поэтому набор

$$
F_{\sigma}(x)=\int \psi_{\Lambda}\left(q^{\prime}, \lambda\right) D_{q \bar{q}^{\prime}}^{\lambda}\left(x^{-1}\right) d \mu(q), \quad \sigma=(q, \lambda, \Lambda),
$$

образует базис решений уравнения (26), причем неизвестные функции $\psi_{\Lambda}\left(q^{\prime}, \lambda\right)$ находятся из уравнения на К-орбите

$$
-H\left(l\left(q^{\prime}, \lambda\right)\right) \psi_{\Lambda}\left(q^{\prime}, \lambda\right)=\Lambda^{2} \psi_{\Lambda}\left(q^{\prime}, \lambda\right)
$$

и как собственные функции оператора $H\left(l\left(q^{\prime}, \lambda\right)\right)$ удовлетворяют условию ортогональности

$$
\int_{Q} \psi_{\Lambda}^{\dagger}(q, \lambda) \psi_{\Lambda^{\prime}}(q, \lambda) d \mu(q)=\delta\left(\Lambda, \Lambda^{\prime}\right)
$$

Данное соотношение является условием нормировки функции $\psi_{\Lambda}\left(q^{\prime}, \lambda\right)$ и определением меры по собственным значениям $\Lambda$.

Таким образом, базис решений уравнения (23), нумеруемый индексом $\sigma$, имеет вид

$$
\Psi_{\sigma}(x, t)=f_{\Lambda}(t) F_{\sigma}(x),
$$


где $f_{\Lambda}(t)$ определяется выражением $(25)$, а $F_{\sigma}(x)$ выражается через решения $\psi_{\Lambda}(\bar{q}, \lambda)$ уравнения (28) по формуле (27). При выполнении условия

$$
\frac{\operatorname{dim} L+\operatorname{ind} L}{2}=1
$$

уравнение (28) сводится к обыкновенному алгебраическому уравнению. В этом случае будем говорить, что исходное уравнение (26) на группе Ли $G$ интегрируемо.

Изложенный выше метод интегрирования примени́м также и в случае однородного пространства $P^{p+1}=\mathbb{R}^{1} \times P^{p}$ с нулевым дефектом и $G$-инвариантной метрикой. Метрический тензор должен быть выбран в виде $\Theta_{\tilde{A} \widetilde{B}}=\left(-1 \otimes-\gamma_{\tilde{A} \widetilde{B}}\right), \tilde{A}, \widetilde{B}=0, \ldots, p$.

Путем аналогичных вычислений легко показать, что набор, образующий базис решений уравнения Клейна-Гордона на $P^{p+1}$, имеет вид

$$
\varphi_{\sigma}(y, t)=\frac{1}{\sqrt{2 \omega}} e^{-i \omega t} \int \psi\left(q^{\prime}, \lambda\right) D_{q \bar{q}^{\prime}}^{\lambda}\left(x^{-1}\right) d \mu\left(q^{\prime}\right), \quad \sigma=(q, \lambda),
$$

где $\omega^{2}=\Lambda^{2}+m^{2}+\tilde{\zeta} \widehat{R}, \tilde{\zeta}=(p-1) /(4 p)$, а функция $\psi\left(q^{\prime}, \lambda\right)$ определяется из системы уравнений (21).

\section{5. ВАКУУМНЫЕ СРЕДНИЕ ТЭИ НА ГРУППАХ ЛИ И ОДНОРОДНЫХ ПРОСТРАНСТВАХ}

Рассмотрим метрический ТЭИ, построенный путем варьирования действия поля по метрике [1]:

$$
\delta S=\int \sqrt{|G|} T^{i j} \delta \Theta_{i j}(g) d \mu(g), \quad S=\int \mathcal{L}^{0}(g) d \mu(g),
$$

где $T_{i j}$ - компоненты ТЭИ в локальных координатах на $M, i, j=0,1, \ldots, n$. Приведем явное выражение для ТЭИ в инвариантном относительно выбора локальных координат виде:

$$
\begin{aligned}
T\left(\eta_{X}, \eta_{Y}\right)\{\bar{\varphi}, \varphi\}=(1 & -2 \zeta) \eta_{X} \bar{\varphi} \eta_{Y} \varphi+\left(2 \zeta-\frac{1}{2}\right) \frac{1}{\sqrt{|\Theta|}} \Theta\left(\eta_{X}, \eta_{Y}\right) \mathcal{L}^{0}- \\
& \left.-\zeta\left[\left(\nabla_{\eta_{X}} \nabla_{\eta_{Y}} \bar{\varphi}\right) \varphi+\bar{\varphi}\left(\nabla_{\eta_{X}} \nabla_{\eta_{Y}} \varphi\right)\right]\right)-\zeta R\left(\eta_{X}, \eta_{Y}\right)|\varphi|^{2}
\end{aligned}
$$

где $X, Y \in \mathbb{R} \otimes L$. Легко перейти к локальным координатам:

$$
T_{i j}(x, t)=T_{A^{\prime} B^{\prime}} \sigma_{i}^{A^{\prime}}(x, t) \sigma_{j}^{B^{\prime}}(x, t), \quad T_{A^{\prime} B^{\prime}} \equiv T\left(\eta_{e_{A^{\prime}}}, \eta_{e_{B^{\prime}}}\right) .
$$

Проведем процедуру квантования скалярного поля с лагранжианом (22). Разложим поле $\phi(x, t)$ по базису решений уравнения $(23)$ :

$$
\hat{\phi}(x, t)=\int d \mu(\sigma)\left[\varphi(x, t) \hat{a}_{\sigma}+\overline{\varphi(x, t)} \hat{a}_{\sigma}^{\dagger}\right],
$$

где $\hat{a}_{\sigma}^{\dagger}, \hat{a}_{\sigma}$ - операторы рождения и уничтожения соответственно, и наложим коммутационные соотношения

$$
\left[\widehat{\varphi}(x, t), \hat{\eta}_{0} \varphi\left(x, t^{\prime}\right)\right]=i \delta\left(x, x^{\prime}\right)
$$


Вакуумные средние ТЭИ определяются выражением

$$
\left\langle\widehat{T}\left(\eta_{X}, \eta_{Y}\right)\right\rangle=\int T\left(\eta_{X}, \eta_{Y}\right)\left\{\varphi_{\sigma}, \bar{\varphi}_{\sigma}\right\} d \mu(\sigma), \quad X, Y \in \mathbb{R}^{1} \otimes L
$$

Подставив в него формулу (30) и воспользовавшись свойствами обобщенных функций $D_{q \bar{q}^{\prime}}^{\lambda}(x)$, получим

$$
\begin{aligned}
\left\langle\widehat{T}\left(\eta_{X}, \eta_{Y}\right)\right\rangle=- & \frac{1}{2} \int \frac{1}{\omega} \overline{\psi_{\Lambda}(q, \lambda)}\left(\frac{1}{2}\left\{l_{X}, l_{Y}\right\}_{+}+\zeta \mathbf{R}(X, Y)\right) \psi_{\Lambda}(q, \lambda) \times \\
& \times d \mu(q) d \mu(\lambda) d \mu(\Lambda),
\end{aligned}
$$

где набор функций $\psi_{\Lambda}(q, \lambda)$ определяется из уравнения $(28)$ и нормирован согласно $(29)$, причем в выражении (34) необходимо учесть, что $l_{e_{0}}=l_{0}=-i \omega$. В тетрадных компонентах $T_{A^{\prime} B^{\prime}}$ выражение (34) может быть записано в виде

$$
\begin{aligned}
\left\langle\widehat{T}_{00}\right\rangle= & \frac{1}{2} \int \omega\left|\psi_{\Lambda}(q, \lambda)\right|^{2} d \mu(q) d \mu(\lambda) d \mu(\Lambda) \\
\left\langle\widehat{T}_{0 A}\right\rangle= & \frac{i}{2} \int \overline{\psi_{\Lambda}(q, \lambda)} l_{A}(q, \lambda) \psi_{\Lambda}(q, \lambda) d \mu(q) d \mu(\lambda) d \mu(\Lambda) \\
\left\langle\widehat{T}_{A B}\right\rangle=- & \frac{1}{2} \int \frac{1}{\omega} \overline{\psi_{\Lambda}(q, \lambda)}\left(\frac{1}{2}\left\{l_{A}(q, \lambda), l_{B}(q, \lambda)\right\}_{+}+\zeta R_{A B}\right) \times \\
& \times \psi_{\Lambda}(q, \lambda) d \mu(q) d \mu(\lambda) d \mu(\Lambda) .
\end{aligned}
$$

Достаточно рассчитать для выбранной группы Ли $G \lambda$-представление ее алгебры Ли $L$, чтобы проинтегрировать уравнение (28) и сразу получить явные выражения для вакуумных средних ТЭИ.

Рассмотрим частный случай, когда нет необходимости решать уравнение (28), а именно когда на группе Ли $M$ существует биинвариантная метрика

$$
\left\langle\operatorname{Ad}_{g} X \operatorname{Ad}_{g} Y\right\rangle=\langle X, Y\rangle, \quad X, Y \in \mathbb{R}^{1} \otimes L .
$$

Действительно, из соотношения

$$
\xi_{X}(g)=-\operatorname{Ad}_{X}^{A^{\prime}}(g) \eta_{A^{\prime}}(g)
$$

и определения (38) следует $H(\xi)=H(\eta)$ и, следовательно, $H\left(l\left(q^{\prime}\right)\right)=\Lambda^{2}=$ const. Вакуумные средние ТЭИ примут вид

$$
\begin{aligned}
\left\langle T\left(\eta_{X}, \eta_{Y}\right)\right\rangle=- & \frac{1}{2} \int \frac{1}{2 \omega}\left(\int\left\{l_{X}(q, \lambda), l_{Y}(q, \lambda)\right\}_{+} \delta(q, \bar{q}) d \mu(q)+\right. \\
& +\zeta \mathbf{R}(X, Y)) \chi_{\lambda}(0) d \mu(\lambda),
\end{aligned}
$$

где $\chi_{\lambda}(0)=\int D_{q \bar{q}}^{\lambda}(0) d \mu(q)$ - обобщенный характер представления (14) группы Ли $G$. В тетрадных компонентах выражение (39) примет вид

$$
\left\langle\widehat{T}_{00}\right\rangle=\frac{1}{2} \int \omega \chi_{\lambda}(0) d \mu(\lambda), \quad\left\langle\widehat{T}_{0 A}\right\rangle=0,
$$




$$
\left\langle\widehat{T}_{A B}\right\rangle=-\frac{1}{2} \int \frac{1}{2 \omega}\left(\int\left\{l_{A}(q, \lambda), l_{B}(q, \lambda)\right\}_{+} \delta(q, \bar{q}) d \mu(q)+\zeta R_{A B}\right) \chi_{\lambda}(0) d \mu(\lambda) .
$$

Заметим, что биинвариантная метрика (38) существует тогда и только тогда, когда группа Ли $G$ унимодулярна: $\operatorname{det}\left(\operatorname{Ad}_{g}\right)=1, g \in G$, причем достаточным условием существования метрики (38) является компактность группы Ли $G$; при этом характер представления $\chi(0, \lambda)$ имеет конечное значение.

Рассмотрим задачу вычисления вакуумных средних ТЭИ на однородном пространстве $P^{p+1}$. Пусть $T_{i j}$ - локальные координаты ТЭИ скалярного поля на $P^{p+1}$, $i, j=0, \ldots, p$. Введем "квазитетрадные" компоненты ТЭИ

$$
T_{\tilde{A} \widetilde{B}}(g) \equiv T_{i j}(g) \eta_{\tilde{A}}^{i}(y, h) \eta_{\widetilde{B}}^{j}(y, h)
$$

В силу соотношений $\eta_{\tilde{A}}^{i} \sigma_{j}^{\tilde{A}}=\delta_{j}^{i}, \eta_{\tilde{A}}^{i} \sigma_{i}^{\widetilde{B}}=\delta_{\tilde{A}}^{\widetilde{B}}$ всегда можно перейти от величин (40) к локальным координатам и обратно. В случае, если $\operatorname{dim} H=0$, т.е. $P$ - группа Ли, величины (40) являются константами и тетрадными компонентами тензорного поля $T$.

Воспользовавшись свойствами обобщенных функций $D_{q \bar{q}^{\prime}}^{\lambda}(x)$, для “квазитетрадных" компонент вакуумных средних ТЭИ получим

$$
\begin{aligned}
\left\langle\widehat{T}_{00}\right\rangle & =-\frac{1}{2} \int \omega|\psi(q, \lambda)|^{2} d \mu(q) d \mu(\lambda), \\
\left\langle\widehat{T}_{0 a}\right\rangle & =\frac{i}{2} \int \overline{\psi(q, \lambda)}\left(l_{a}(q, \lambda) \psi(q, \lambda)\right) d \mu(q) d \mu(\lambda), \\
\left\langle\widehat{T}_{a b}\right\rangle & =\int \frac{1}{2 \omega} \overline{\psi(q, \lambda)}\left(\left\{l_{a}, l_{b}\right\}_{+}-\tilde{\zeta} R_{a b}\right) \psi(q, \lambda) d \mu(q) d \mu(\lambda),
\end{aligned}
$$

где $l_{a}=l_{a}(q, \lambda)$ - операторы $\lambda$-представления $s_{P}$-типа, соответствующие однородному пространству (см. формулу $(20)), \psi(q, \lambda)$ - решение системы уравнений $(21)$.

\section{6. ПЕРЕНОРМИРОВКА ТЭИ НА ГРУППАХ ЛИ ПРИ ПОМОЩИ ОБОБЩЕННОЙ К-ФУНКЦИИ}

Вакуумные средние ТЭИ скалярного поля $\varphi(x, t)$ могут быть получены вариацией по метрике однопетлевого эффективного действия $W$ :

$$
\left\langle T_{i j}\right\rangle=-\frac{2}{\sqrt{|\Theta(g)|}} \frac{\delta W}{\delta \Theta_{i j}}, \quad i, j=1, \ldots, n .
$$

Эффективное действие $W$ связано с оператором действия квантового поля при помощи вариационного принципа Швингера $W=\langle\delta \widehat{S}\rangle$ и содержит в себе все расходимости теории. Для скалярного поля с лагранжианом (22) эффективное действие $W$ на $M^{n}$ имеет вид

$$
W=i \ln \int D[\varphi] e^{i S}=\frac{i}{2} \ln \operatorname{det}\left(\frac{i \widehat{F}}{2 \pi \mu^{2}}\right), \quad \widehat{F} \equiv \partial_{t}^{2}-\Delta_{G}+\zeta R+m^{2},
$$

где $\mu$ - нормировочная константа, не зависящая от метрики и имеющая размерность массы. 
Рассмотрим обобщенную $\zeta$-функцию оператора $\widehat{F}$

$$
\zeta(s)=\int \lambda_{\sigma}^{-s} d \mu(\sigma)
$$

где $\lambda_{\sigma}$ - собственные значения оператора $\widehat{F}$. В случае дискретности спектра оператора $\widehat{F}$ обобщенная $\zeta$-функция сводится к обычной $\zeta$-функции Римана. Обобщенная $\zeta$-функция $\zeta(s)$ допускает аналитическое продолжение в комплексную плоскость, регулярное в точке $s=0$, и может быть определена как интеграл по $M$ от локальной ל-функции:

$$
\zeta(g, s)=\int \lambda_{\sigma}^{-s} \overline{\varphi_{\sigma}(x, t)} \varphi_{\sigma}(x, t) d \mu(\sigma), \quad \zeta(s)=\int \zeta(g, s) d \mu(g),
$$

где $\varphi_{\sigma}(x, t)$ - полный и ортогональный набор собственных функций оператора $\widehat{F}$. Уравнение $\widehat{F} \varphi_{\sigma}(x, t)=\lambda_{\sigma} \varphi_{\sigma}(x, t)$ сводится к уравнению $(28)$, где $\varphi_{\sigma}(x, t)$ определяется соотношением (26) с условием нормировки (24) и собственным значением $\lambda_{\sigma}=\Lambda^{2}-\omega^{2}+m^{2}+\zeta R$.

Регуляризованное эффективное действие $W$ выражается через обобщенную $\zeta$-функцию

$$
W(s)=-\frac{i}{2}\left(\zeta^{\prime}(s)+\zeta(s) \ln \left(-2 \pi i \mu^{2}\right)\right), \quad W_{\text {ren }}=\left.W(s)\right|_{s=0},
$$

и уже не содержит расходимостей. Перенормированный ТЭИ выражается через $W_{\text {ren }}$ согласно формуле (41).

Основную трудность представляет собой вычисление функциональных производных эффективного действия $W_{\text {ren }}$ по метрике, так как для этого необходимо, вообще

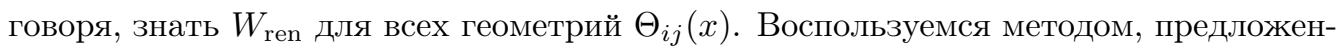
ным в работе [17], когда сначала вычисляются функциональные производные по $\Theta_{i j}(x)$ от эффективного действия, а уже потом берется аналитическое продолжение при $s=0$ :

$$
\left\langle\widehat{T}_{i j}\right\rangle_{\mathrm{ren}}=-\left.\frac{1}{\sqrt{|\Theta(x)|}} \frac{\delta W(s)}{\delta \Theta_{i j}}\right|_{s=0} .
$$

Подставив сюда выражение (44), получим

$$
\left\langle T_{i j}\right\rangle_{\mathrm{ren}}=-\frac{i}{2}\left(\left.\frac{d}{d s}\right|_{s=0} Z_{i j}(g, s)+Z_{i j}(g, 0) \ln \left(-2 \pi i \mu^{2}\right)\right),
$$

где функция $Z_{i j}(g, s)$ представляет собой аналитическое продолжение по переменной $s$ вариации $\zeta$-функции $\zeta(s)$ по метрике:

$$
Z_{i j}(g, s)=-\frac{2}{\sqrt{|\Theta|}} \frac{\delta \zeta(s)}{\delta \Theta^{i j}},
$$

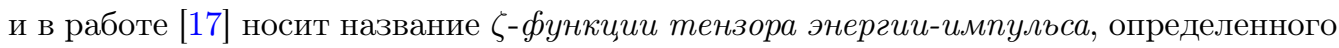
формулой (41). Из соотношения (46) следует, что вычисление перенормированного значения ТЭИ сводится к поиску аналитического продолжения по $s=0$ производных от функции $Z_{i j}(g, s)$. 
Так как

$$
S\left[\overline{\varphi_{\sigma}}, \varphi_{\sigma}\right]=-\frac{1}{2} \int \overline{\varphi_{\sigma}} \widehat{F} \varphi_{\sigma} d \mu(\sigma)=-\frac{1}{2} \int \lambda_{\sigma}|\varphi(x, t)|^{2} d \mu(x) d t
$$

и собственные функции $\varphi_{\sigma}(x, t)$ удовлетворяют условию ортогональности, то оказывается возможным вычислить вариацию собственного значения $\lambda_{\sigma}$ :

$$
\frac{\delta \lambda_{\sigma}}{\delta \Theta^{i j}}=-2 \frac{\delta S\left[\overline{\varphi_{\sigma}}, \varphi_{\sigma}\right]}{\delta \Theta^{i j}}=-\frac{1}{2} \sqrt{|\Theta| \Theta_{i j}} \lambda_{\sigma}\left|\varphi_{\sigma}\right|^{2}-2 \frac{\delta S\left[\overline{\varphi_{\sigma}}, \varphi_{\sigma}\right]}{\delta \Theta^{i j}} .
$$

Данное равенство позволяет выразить $Z_{i j}(g, s)$ через локальную $\zeta$-функцию (43) и функциональную производную действия $S$ по метрике:

$$
Z_{i j}(g, s)=2 s \zeta_{i j}(g, s+1)-s \zeta(g, s) \Theta_{i j}, \quad \zeta_{i j}(g, s) \equiv \int \lambda_{\sigma}^{-s} T_{i j}\left\{\overline{\varphi_{\sigma}}, \varphi_{\sigma}\right\} d \mu(\sigma) .
$$

Так как мы рассматриваем ситуацию, когда применение метода обобщенной $\zeta$-функции позволяет регуляризовать эффективное действие $W_{\text {ren }}$, то величины $\zeta(g, 0)$ и $\zeta^{\prime}(g, 0)$ должны быть конечными и, следовательно,

$$
\lim _{s \rightarrow 0} s \zeta^{\prime}(g, s)=0, \quad \lim _{s \rightarrow 0} s \zeta(g, s)=0 .
$$

Учитывая соотношения (48), подставим выражение (47) в формулу (46) и после ряда вычислений получим выражение для перенормированного ТЭИ на $M$ :

$$
\begin{aligned}
\left\langle T_{A^{\prime} B^{\prime}}\right\rangle_{\mathrm{ren}}=- & i \zeta_{A^{\prime} B^{\prime}}(1)+i \frac{1}{2} \Theta_{A^{\prime} B^{\prime}} \zeta(g, 0)- \\
& \quad-\left.i s\left[\zeta_{A^{\prime} B^{\prime}}^{\prime}(s+1)+\zeta_{A^{\prime} B^{\prime}}(s+1) \ln \left(-2 \pi i \mu^{2}\right)\right]\right|_{s=0},
\end{aligned}
$$

где

$$
\begin{gathered}
\zeta_{A^{\prime} B^{\prime}}(s)=\int \lambda_{\sigma}^{-s} T_{A^{\prime} B^{\prime}}\left\{\overline{\varphi_{\sigma}}, \varphi_{\sigma}\right\} d \mu(\sigma)= \\
=\overline{\zeta_{A^{\prime} B^{\prime}}(s)}+\frac{1}{2} \Theta_{A^{\prime} B^{\prime}} \zeta(g, s-1)-\zeta R_{A^{\prime} B^{\prime}} \zeta(g, s), \\
\overline{\zeta_{A^{\prime} B^{\prime}}(s)}=\int \lambda_{\sigma}^{-s} \eta_{\left\{A^{\prime}\right.} \overline{\varphi_{\sigma}} \eta_{\left.B^{\prime}\right\}} \varphi_{\sigma} d \mu(\sigma), \quad \zeta(g, s)=\int \lambda_{\sigma}^{-s}\left|\varphi_{\sigma}(x, t)\right|^{2} d \mu(\sigma) .
\end{gathered}
$$

Собственные функции оператора $\widehat{F}$ будем искать в виде набора (30). Тогда

$$
\begin{aligned}
\overline{\zeta_{A^{\prime} B^{\prime}}(s)} & =-\frac{1}{2} \int \lambda_{\Lambda \lambda}^{-s} \overline{\psi_{\Lambda}(q, \lambda)}\left\{l_{A^{\prime}}, l_{B^{\prime}}\right\}_{+} \psi(q, \lambda) d \mu(q) d \mu(\lambda) d \mu(\Lambda), \\
\zeta(g, s) & =\int \lambda_{\Lambda \lambda}^{-s}|\psi(q, \lambda)|^{2} d \mu(q) d \mu(\lambda) d \mu(\Lambda) .
\end{aligned}
$$

Из формулы (49) следует, что предел выражения $s \zeta_{A^{\prime} B^{\prime}}(s+1)$ при $s \rightarrow 0$ должен быть конечным:

$$
\alpha_{A^{\prime} B^{\prime}}=\lim _{s=0} s \zeta_{A^{\prime} B^{\prime}}(s+1) .
$$

Следовательно,

$$
\zeta_{A^{\prime} B^{\prime}}(s+1) \sim \frac{\alpha_{A^{\prime} B^{\prime}}}{s}, \quad s \zeta_{A^{\prime} B^{\prime}}^{\prime}(s+1) \sim-\frac{\alpha_{A^{\prime} B^{\prime}}}{s} .
$$


Рассмотрим асимптотическое разложение для недиагональных элементов ядра теплопроводности $H\left(t, g, g^{\prime}\right)$ :

$$
H\left(t, g, g^{\prime}\right)-i \sim \frac{e^{-i \beta\left(g, g^{\prime}\right) /(2 t)}}{(4 \pi t)^{2}} \sum_{k=0}^{\infty} a_{k}\left(g, g^{\prime}\right) t^{k}, \quad t \rightarrow 0
$$

где $\beta\left(g, g^{\prime}\right)$ - половина квадрата длины геодезической между точками $g$ и $g^{\prime}, a_{k}\left(g, g^{\prime}\right)-$ коэффициенты ядра теплопроводности, выражающиеся через кривизну и метрику многообразия $M$. В нашем случае скалярная кривизна $R$ не зависит от локальных координат, и при совпадающих аргументах $g=g^{\prime}$ в размерности $n=3$ мы будем иметь

$$
a_{1}=\left(\frac{1}{6}-\zeta\right) R, \quad a_{2}=\frac{1}{180}\left(R^{A B C D} R_{A B C D}-R^{A B} R_{A B}\right)+\frac{1}{2} a_{1}^{2} .
$$

Связь между ядром теплопроводности и локальной $\zeta$-функцией выражается равенством

$$
\zeta\left(s, g, g^{\prime}\right)=\frac{i}{\Gamma(s)} \int_{0}^{\infty} d t(i t)^{s-1} H\left(t, g, g^{\prime}\right), \quad \zeta(s, g)=\lim _{g^{\prime} \rightarrow g} \zeta\left(s, g, g^{\prime}\right)
$$

На основе соотношений (53) и (54) при $m=0$ в работе [17] исследовалась структура полюсов функции $\zeta_{i j}(s+1, g)$ и было получено явное выражение для величины $\alpha_{i j}$ через коэффициенты $a_{k}(g)$ ядра теплопроводности (53). Для нашего случая группового многообразия $M$ будем иметь

$$
\alpha_{A^{\prime} B^{\prime}}=\frac{1}{(4 \pi)^{2}} \Theta_{A^{\prime} B^{\prime}} a_{2} .
$$

Отсюда следует, что при $a_{2}=0$ для функции $\zeta_{A^{\prime} B^{\prime}}(s+1)$ выполняются соотношения, аналогичные (48), для локальной $\zeta$-функции:

$$
s \zeta_{A^{\prime} B^{\prime}}(s+1)=0, \quad s \zeta_{A^{\prime} B^{\prime}}^{\prime}(s+1)=0, \quad s \rightarrow 0 .
$$

Выражение для перенормированного ТЭИ принимает более простой вид:

$$
\left\langle\widehat{T}_{A^{\prime} B^{\prime}}\right\rangle_{\text {ren }}=-\left.i\left(\overline{\zeta_{A^{\prime} B^{\prime}}(s+1)}-\zeta R_{A^{\prime} B^{\prime}} \zeta(g, s+1)\right)\right|_{s=0},
$$

и не зависит от параметра $\mu$. Сравним выражение (55) с известной формулой для следа расходящейся части вакуумного среднего ТЭИ: $\left\langle T_{i}^{i}\right\rangle_{\operatorname{div}}=-a_{2} /\left(16 \pi^{2}\right)$. Тогда

$$
\alpha_{A^{\prime} B^{\prime}}=-\Theta_{A^{\prime} B^{\prime}}\left\langle T_{i}^{i}\right\rangle_{\mathrm{div}} .
$$

Для безмассового конформно-инвариантного поля в четырехмерном пространстве-времени $(\zeta=1 / 6)$ след ТЭИ равен нулю, и, следовательно, след вакуумного среднего $\left\langle T_{i}^{i}\right\rangle$ также равен нулю. Так как $\left\langle T_{i}^{i}\right\rangle_{\mathrm{ren}}=\left\langle T_{i}^{i}\right\rangle-\left\langle T_{i}^{i}\right\rangle_{\mathrm{div}}$, то след перенормированного ТЭИ в общем случае отличен от нуля (имеет место так называемая конформная аномалия).

Таким образом, при $m=0$ и $\zeta=1 / 6$ выполнение соотношений (56) эквивалентно отсутствию конформной аномалии для ТЭИ скалярного поля. 


\section{7. ЭФФЕКТ КАЗИМИРА НА ГРУППЕ ЛИ $\mathbb{R}^{1} \times E(2)$}

Рассмотрим групповое многообразие $M=\mathbb{R} \times E(2)$, где $G=E(2)$ - группа движений двумерной плоскости. Групповое многообразие группы $G$ обладает топологией цилиндра $\mathbb{R}^{2} \times \mathbb{S}^{1}$. Трехмерная алгебра Ли $\mathfrak{e}(2)$ группы Ли $E(2)$ определяется коммутационными соотношениями

$$
\left[e_{2}, e_{3}\right]=e_{1}, \quad\left[e_{3}, e_{1}\right]=-e_{2}, \quad \epsilon>0 .
$$

Единственная функция Казимира $K(f)=f_{1}^{2}+f_{2}^{2}$ алгебры $\mathfrak{e}(2)$ определяет невырожденные К-орбиты $\mathcal{O}_{\lambda}=\left\{f \in \mathbb{R}^{3} \mid K(f)=\lambda\right\}$, каждая из которых проходит через параметризованный ковектор $\lambda=(j, 0,0), j>0$. Операторы $\lambda$-представления имеют вид

$$
l_{1}=i j \cos q, \quad l_{2}=-i j \sin q, \quad l_{3}=\partial_{q} .
$$

Из условия вещественности функций перехода к каноническим координатам Дарбу на $\mathrm{K}$-орбите следует, что $Q=[0,2 \pi)$. Операторы $l_{A}$ косоэрмитовы относительно меры $d q$. Спектральная мера оператора Казимира есть $d \mu(\lambda)=j d j /(2 \pi)^{2}$. Так как группа Ли $E(2)$ содержит компактную подгруппу $S O(2)$ с алгеброй $\left\{e_{3}\right\}$, то $\mathrm{K}$-орбиты $\mathcal{O}_{\lambda}(j)$ квантуются, и параметр $j$ принимает целочисленные значения.

При помощи группы автоморфизмов алгебры Ли $\mathfrak{e}(2)$ правоинвариантную метрику $\gamma^{a b}$ на $E(2)$ можно привести к диагональному виду $\gamma^{a b}=\operatorname{diag}(A, B, C)$. Тогда ненулевые компоненты тензора Риччи на $M$ суть

$$
R_{11}=-R_{22}=\frac{A^{2}-B^{2}}{2 A^{2} B} C, \quad R_{33}=\frac{(A-B)^{2}}{2 A B} .
$$

При преобразовании (27) оператор Лапласа на $E(2)$ перейдет в оператор

$$
H(l(q, \lambda))=\gamma^{A B} l_{A}(q, \lambda) l_{B}(q, \lambda)=C \frac{d^{2}}{d q^{2}}+(B-A) j^{2} \cos q^{2}-B j^{2}
$$

на $\mathrm{K}$-орбите, зависящий от переменной $q$ и параметра $j$. Решение уравнения Клейна-Фока (28) будем искать в виде периодических функций Матье:

$$
\psi_{n}(q)=c e_{n}\left(\frac{A-B}{4 C} j^{2}, q\right), \quad n \in \mathbb{Z} .
$$

Тогда для спектра получаем условие

$$
\Lambda_{n}^{2}=C a_{n}\left(\frac{A-B}{4 C} j^{2}\right)+\frac{A+B}{2} j^{2},
$$

где $a_{n}$ - собственные функции уравнения Матье. Для собственных значений оператора $\widehat{F}=\square+m^{2}+\zeta R$ имеем

$$
\lambda_{\sigma}=\Lambda_{n}^{2}-j_{0}^{2}+m^{2}+\zeta R, \quad \sigma=\left(n, j, j_{0}\right) .
$$

Меры по $d \mu\left(j_{0}\right)$ и $d \mu(\Lambda)$ выберем в виде

$$
\int f\left(j_{0}, n\right) d \mu\left(j_{0}\right) d \mu(\Lambda)=\frac{1}{2 \pi^{2}} \sum_{n=0}^{\infty} \int_{0}^{\infty} f\left(j_{0}, n\right) d j_{0} .
$$


По формуле (34) могут быть рассчитаны неперенормированные вакуумные средние ТЭИ. Они имеют довольно громозкий вид и в настоящей работе не приводятся. Найдем перенормированное значение плотности энергии $\left\langle\widehat{T}_{00}\right\rangle_{\mathrm{ren}}$.

Рассмотрим локальную $\zeta$-функцию оператора $\widehat{F}$

$$
\zeta(s)=\frac{\sqrt{A B C}}{(2 \pi)^{3}} \sum_{n=0}^{\infty} \int_{0}^{\infty} j d j \int_{0}^{\infty} d j_{0} \lambda_{\sigma}^{-s} .
$$

Воспользуемся интегральным преобразованием

$$
\lambda^{-s}=\frac{1}{\Gamma(s)} \int_{0}^{\infty} t^{s-1} e^{-z t} d t, \quad \operatorname{Re} s>0, \quad \operatorname{Re} z>0,
$$

и представим $\zeta(s)$ в виде

$$
\zeta(s)=-i \frac{\sqrt{\pi A B C}}{2(2 \pi)^{3} \Gamma(s)} \int_{0}^{\infty} d t t^{s-3 / 2} e^{-t\left(m^{2}+\zeta R\right)} \sum_{n=0}^{\infty} I_{n}(t),
$$

где

$$
I_{n}(t)=\int_{0}^{\infty} j d j \exp \left(-t\left[C a_{n}\left(\frac{A-B}{4 C} j^{2}\right)+\frac{A+B}{2} j^{2}\right]\right) .
$$

Интеграл (59) в общем случае $A \neq B$ не выражается через элементарные функции, но возможно получить его асимптотическое поведение:

$$
I_{1}(t) \sim \frac{2 e^{-C t}}{(3 A+B) t}, \quad I_{n}(t) \sim \frac{e^{-C t n^{2}}}{(A+B) t}, \quad t \rightarrow \infty, \quad n \neq 1 .
$$

Тогда для $\zeta(s)$ получаем

$$
\begin{aligned}
& \zeta(s)=- i \frac{\sqrt{\pi A B C}}{2(2 \pi)^{3}(A+B)} \frac{\Gamma(s-3 / 2)}{\Gamma(s)}\left[C^{-(s-3 / 2)} E_{1}^{\left(m^{2}+\zeta R\right) / C}\left(s-\frac{3}{2}, 1\right)+\right. \\
&\left.+\left(m^{2}+\zeta R\right)^{-(s-3 / 2)}-\frac{A-B}{3 A+B}\left(C+m^{2}+\zeta R\right)^{-(s-3 / 2)}\right],
\end{aligned}
$$

где $E_{1}^{c^{2}}(s, 1) \equiv \sum_{n=1}^{\infty}\left(n^{2}+c^{2}\right)^{-s}-\zeta$-функция Эпштейна-Гурвица. Согласно выражению (49) получим

$$
\left\langle\widehat{T}_{00}\right\rangle_{\mathrm{ren}}=\left.\frac{i}{2} \zeta(s)\right|_{s=0}
$$

Используя формулу Абеля-Плана для аналитического продолжения $\zeta$-функции Эпштейна-Гурвица, имеем:

$$
E_{1}^{c^{2}}\left(-\frac{3}{2}, 1\right)=\frac{1}{2} c^{3}+2 i \int_{c}^{\infty} \frac{\left(c^{2}-t^{2}\right)^{3 / 2}}{e^{2 \pi t}-1} d t .
$$

Тогда

$$
\begin{aligned}
\left\langle\widehat{T}_{00}\right\rangle_{\mathrm{ren}}= & \frac{\sqrt{A B C}}{32 \pi^{2}(A+B)}\left[2 \int_{\sqrt{\left(m^{2}+\zeta R\right) / c}}^{\infty} \frac{\left[C t^{2}-m^{2}-\zeta R\right]^{3 / 2}}{e^{2 \pi t}-1} d t+\right. \\
& \left.+\frac{1}{2}\left(m^{2}+\zeta R\right)^{3 / 2}-\frac{A-B}{3 A+B}\left(C+m^{2}+\zeta R\right)^{3 / 2}\right] .
\end{aligned}
$$


Выражение (61) для плотности энергии является ненулевым, и, следовательно, имеет место эффект поляризации вакуума скалярного поля на $M$. При $A=B$ точное значение $I_{n}(t)$ совпадает с формулой (60), и в случае безмассового поля $m=0$ для вакуумных средних ТЭИ скалярного поля получаем $(A=B=1)$

$$
\left\langle\widehat{T}_{A^{\prime} B^{\prime}}\right\rangle_{\text {ren }}=\frac{C^{3 / 2}}{1440 \pi^{2}} \operatorname{diag}\left(-1,1,1,-\frac{3}{C}\right) .
$$

Случай $A=B$ соответствует плоской метрике, и выражение (62) описывает хорошо известный эффект Казимира на $\mathbb{R}^{2} \times \mathbb{S}^{1}$ (см. книгу [1]).

Благодарности. Работа выполнена при частичной финансовой поддержке Аналитической ведомственной целевой программы "Развитие научного потенциала высшей школы" Министерства образования и науки РФ (гранты № 2.1.1/3436, 2.1.1/12999), Федеральной целевой программы "Научные и научно-педагогические кадры инновационной России” Министерства образования и науки РФ (гранты № 02.740.11.0238, П691, П789).

\section{Список литературы}

[1] А.А. Гриб, С.Г. Мамаев, В.М. Мостепаненко, Квантовые эффекты в интенсивных внешних полях, Атомиздат, М., 1980.

[2] Н. Биррелл, П. Девис, Квантованные поля в искривленном пространстве-времени, Мир, М., 1984.

[3] S. A. Fulling, Aspects of Quantum Field Theory in Curved Space-Time, London Math. Soc. Stud. Texts, 17, Cambridge Univ. Press, Cambridge, 1989.

[4] A. DeBenedictis, K.S. Viswanathan, Stress-Energy Tensors for Higher Dimensional Gravity, arXiv: hep-th/9911060.

[5] S. M. Christensen, Phys. Rev. D, 14:10 (1976), 2490-2501.

[6] Я.Б. Зельдович, А. А. Старобинский, ЖЭТФ, 61:6 (1971), 2161-2175.

[7] C. Molina-Paris, "Energy-Momentum Tensor and Particle Creation in the de Sitter Universe", The Eighth Marcel Grossmann Meeting, Part A, B (Jerusalem, 1997), eds. T. Piran, R. Ruffini, World Sci. Publ., River Edge, NJ, 1999, 827-829, arXiv: gr-qc/9710088.

[8] R. Camporesi, A. Higichi, Phys. Rev. D, 45:10 (1992), 3591-3603.

[9] А. А. Кириллов, УМН, 17:4(106) (1962), 57-110.

[10] А.А. Кириллов, Функи. анализ и его прил., 2:2 (1968), 40-55.

[11] А.А. Кириллов, Элементы теории представлений, Наука, М., 1978.

[12] B. Kostant, "Quantization and Unitary Representations. I. Prequantization", Lectures in Modern Analysis and Applications, v. III, Lecture Notes in Math., 170, ed. C. T. Taam, Springer, Berlin, 1970, 87-208.

[13] J.-M. Souriau, Structure des systèmes dynamiques. Maîtrises de mathématique, Dunod, Paris, 1970.

[14] А. В. Шаповалов, И. В. Широков, ТМФ, 104:2 (1995), 195-213.

[15] А. В. Шаповалов, И. В. Широков, ТМФ, 106:1 (1996), 3-15.

[16] У. Миллер, Симметрия и разделение переменных, Мир, М., 1981.

[17] V. Moretti, Phys. Rev. D, 56:12 (1997), 7797-7819, arXiv: hep-th/9705060.

[18] И.В. Широков, К-орбиты, гармонический анализ на однородных пространствах и интегрирование дифференииальных уравнений, Препринт, ОмГУ, Омск, 1998.

[19] И. В. Широков, ТМФ, 123:3 (2000), 407-423. 